\title{
Effect of activated carbon from coffee residue on the properties of weft-knitted polyester mattress fabric
}

\begin{abstract}
Activated carbon (AC) from coffee residue was added to polyethylene terephthalate spinning solution to process the polyester (PET) drawn textured (DT) yarn. The antibacterial activity of the weft-knitted mattress fabric was investigated, compared to the PET-DTY and the PET spun yarn. With AC of PET-DTY, 93.1\% antibacterial rate of mattress fabric had been achieved, while that of PET-DTY without AC was $59.2 \%$ and that of spun yarn fabric was $63.1 \%$. For the sample involved AC, the uniform distribution of AC was observed by scanning electronic microscopy (SEM). The $32.77 \%$ heat preservation ratio and $35.97 \mathrm{~cm} 2$ liquid transportability were obtained in this case, which were better than the situations of DTY and spun yarns. There was $13.2 \%$ moderate reduction in breaking strength. No apparent differences were found in the chemical structure by Fourier Transform Infrared Spectroscopy (FTIR), the moisture permeability and the pilling performance of fabrics.
\end{abstract}

Volume 4 Issue 3 - 2018

\author{
Ailan Wan, ${ }^{1,2}$ Gaoming Jiang, ${ }^{1,2}$ Honglian \\ Cong, ${ }^{1,2}$ Xuhong Miao ${ }^{1,2}$ \\ 'Engineering Research Center of Knitting Technology, Jiangnan \\ University, China \\ ${ }^{2}$ Key Laboratory of Eco-textiles, Jiangnan University, China
}

Correspondence: Ailan Wan, Engineering Research Center of Knitting Technology, Key Laboratory of Eco-textiles, Ministry of Education, Jiangnan University, Wuxi 214122, China,

Email ailan.wan@jiangnan.edu.cn

Received: March 21, 2017| Published: June 18, 2018

Keywords: activated carbon, coffee residue, polyester drawn textured yarn, antibacterial property, weft-knitted mattress fabric

Abbreviations: AC, activated carbon; PET, polyester; DT, drawn textured; SEM, scanning electronic microscopy; FTIR, fourier transform infrared spectroscopy; PET-DTY, polyester drawn textured yarn

\section{Introduction}

Antibacterial property of weft-knitted mattress fabric for sleeping and resting has been a serious problem for both industry and consumers. The polyester fibres are the main materials for the mattress fabrics, which have good wear resistance and high performance at low cost. However, the sweat liquid and grease cannot be absorbed and the bacterial may breed gradually. The chemical coating with a resin has been difficult to maintain a durable binding with the mattress fabric surface. A great deal of interest has recently been stimulated by the improvement or even new techniques for processing solid waste into value-added materials.

A promising alternative for the antibacterial activity on mattress fabric is activated carbon (AC) production. Activated carbons (ACs) are obtained from the spent coffee residue as the precursors in a chemical scheme. ${ }^{1,2}$ when activated, the initial materials undergoes partial oxidation in the walls of the pre-existing channels, thus, developing a high surface area, forming $\mathrm{AC}$, a porous carbonaceous material with a non-graphitic crystalline structure would be obtained. ${ }^{3}$ The AC possesses countless applications, such as: gas

Table I The characteristics and specifications of three mattress fabrics storage, ${ }^{1}$ pharmaceutical industry usage, water and air treatment, environmental decontamination processes, ${ }^{4,5}$ catalysis and the food industry. ${ }^{6}$ However, little work has been published on using AC for antibacterial activity of fabrics.

This work studied the effect of AC prepared from spent coffee grounds on antibacterial activity of weft-knitted mattress fabric. The $\mathrm{AC}$ was added into polyethylene terephthalate spinning solution to process the polyester drawn textured yarn (PET-DTY). The surface morphology, chemical groups and physical properties of PET-DTY with and without $\mathrm{AC}$ were investigated. The pilling performance, the heat preservation, moisture permeability and the liquid transportability of mattress fabrics were evaluated as well. The PET spun yarns were used as a reference sample due to their use as one of the main materials in weft-knitted mattress fabrics.

\section{Experimental}

\section{Materials}

In this study, the PET yarn count was 167dtex. All the PET-DTY with or without AC and PET spun yarns were supplied by Taicang Jinhui synthetic fibre industry co., ltd. The mattress fabric structured with net jacquard was knitted by double-faced computer jacquard machine with 38 inch cylinder diameter and 20needles per inch. The characteristics and specifications of mattress fabrics were shown in Table 1.

\begin{tabular}{lllllll}
\hline \multirow{2}{*}{ Sample } & \multicolumn{2}{l}{ Density of right side } & \multicolumn{2}{l}{ Density of reverse side } & Thickness $/ \mathbf{m m}$ & Surface density $/\left(\mathbf{g ~ m}^{-2}\right)$ \\
\cline { 2 - 5 } & PA & PB & PA & PB & & \\
\hline DTY+ AC & 55 & 50 & 55 & 56 & 1.35 & 230.4 \\
DTY & 57 & 50 & 57 & 56 & 230.1 & 5035.2 \\
Spun yarn & 56 & 51 & 57 & 56 & 246.6 & 5447 \\
\hline
\end{tabular}




\section{Antibacterial activity of mattress fabric}

The antibacterial activity was tested according to GB/T 20944.32008 Textiles-Evaluation for antibacterial activity_Part 3: Shake flask method. The Escherichia coli were chosen in the experiment.

\section{Surface morphology and chemical structure of PET- DTY with AC}

All the samples were cleaned by Soxhlet extraction with acetone and grain alcohol for $4 \mathrm{~h}$, air-dried and then rinsed thoroughly in distilled water to remove reagents, inorganic and proteinaceous materials according to GB/T 6977-2008. Then they were conditioned $24 \mathrm{~h}$ in the constant temperature and humidity laboratory at $20 \pm 2^{\circ} \mathrm{C}$, $65 \pm 2 \%$ RH. Scanning Electronic Microscopy (SEM, JSM-5600LV, JOEL, and Japan) images of PET fibres were taken with an $\mathrm{X}-650$ microscope after conductivity coating. A Fourier transform infrared (FTIR) instrument (Nicolet 5700, Continu $\mu \mathrm{m}$ ) was used to analyze the spectra of the samples. Resolution for the infrared spectra was $4 \mathrm{~cm}^{-1}$ and there were 32 scans for each spectrum.

\section{Characterization of yarn shape and yarn tensile property}

3D Rotational microscopy (KH-1000, QUESTAR Co., USA) image of yarn hairiness was captured by an HIROX optical microscope at $50 \times$ magnifications to observe the yarn shape. The tensile strength and elongation at break of PET-DTY with and without AC, as well as spun yarns were measured on the Electronic Single Yarn Strength Tester (Model YG061, Laizhou Electron Instrument Co. Ltd) with a 5N load cell. The test was carried out at $250 \mathrm{~mm} / \mathrm{min}$ constant speed with a sample holder according to GB/T 3916-1997. All measurements were repeated for fifty equally treatment.

\section{Thermal-wet comfort of mattress fabric}

The heat and moisture transfer coefficient, heat transfer property of three mattress fabrics of PET with and without AC, and spun yarn were tested with YG606D flat heat preserve tester according to GB 11048-1989 Textiles - testing method for warmth retention property. The water vapour transmission property was tested with YG601-II computer fabric moisture permeability instrument according to GB/ T12704-91 Fabrics - Determination of water vapour transmission rate-Dish method. Besides, the wettability of mattress fabric was investigated with liquid-drop method since the mattress was often laid horizontally according to JIS L1907-2010 Testing methods for water absorbency of textiles. The wetted area was measured.

\section{Martindale fuzzing-pilling and abrasion method}

The mattress fabrics were cut into $150 \mathrm{~cm} \times 150 \mathrm{~cm}$ pieces and were placed on the Martindale fuzzing-pilling and abrasion meter (YG401E, Ningbo Textile Instrument Factory, and China) according to GB/T 4802.2-2008 Modified Martindale Method. The right-hand sides of samples were abraded with wool samples from 125 to 5000 rubs with $260 \mathrm{~g}$ loading weight. Fuzzing and pilling are assessed according to British Standard 5811:1986, in accordance with a grading range from 5 (no visual change) to 1 (dense fuzzing and/or pilling which cover the specimen).

\section{Results and discussion}

\section{Antibacterial activity of mattress fabric}

The bacterium solution was obtained at $37^{\circ} \mathrm{C}$ with $24 \mathrm{~h}$ standing in the incubator by 10 times dilution method. The data at $10^{4}$ dilution ratio was chosen to evaluate the antibacterial rate. The total number of bacterial colony is added up statistically in Table 2. For the case of PET-DTY with AC, 93.1\% antibacterial rate of mattress fabric is achieved, while that of PET-DTY without AC is $59.2 \%$ and that of spun yarn fabric is $63.1 \%$. The porous structure 1 and high specific surface area are provided by the $\mathrm{AC}$, which can have the excellent absorption capacity. ${ }^{7}$ The bacteria could not survive without the humid environment by controlling surface moisture effectively.

Table $\mathbf{2}$ The results of total number of bacterial colony

\begin{tabular}{|c|c|c|c|c|c|}
\hline \multirow{2}{*}{ Sample } & \multicolumn{4}{|c|}{ Total number of bacterial colony } & \multirow{2}{*}{ The bacteriostatic rates } \\
\hline & $10^{1}$ & $10^{2}$ & $10^{3}$ & $10^{4}$ & \\
\hline Control fabric & $>$ & $>$ & $>300$ & 103 & - \\
\hline DTY with AC & $>$ & $>$ & 17 & 5 & $91.30 \%$ \\
\hline DTY & $>$ & $>$ & 101 & 42 & $59.20 \%$ \\
\hline Spun yarn & $>$ & $>$ & 158 & 38 & $63.10 \%$ \\
\hline
\end{tabular}

Obviously, DTY with AC results in the best antibacterial activity, which can be clearly seen from Figure 1 . Tannic acid in the AC has the bacteriostasis against the Escherichia coli.

To understand why the fabric of DTY with AC produced the best antibacterial activity, SEM and FTIR was used to detect the distribution and chemical changes of AC in PET-DTY, and to measure the cross-section of the PET fibres.

\section{Morphology and chemical structure of PET fibres}

The SEM images (Figure 2) show the surface structure of PETDTY with and without AC, as well as PET spun yarn. Figure 2A presents that the $\mathrm{AC}$ distributes uniformly in the fibres compared to Figure $2 \mathrm{~B}$. Along the fibre axis, the edges are found special-shaped in Figure 2A \& Figure 2B, which is different from the circular section of spun yarn in Figure 2C.

No apparent differences are found in the chemical structure for PET-DTY with and without ACs from FTIR results in Figure 3. The chemical structure is not affected by surface particles AC of DTY fiber.

The surface hairs and tensile strength of yarns will affect the fabric thermal-wet comfort and pilling performance. 


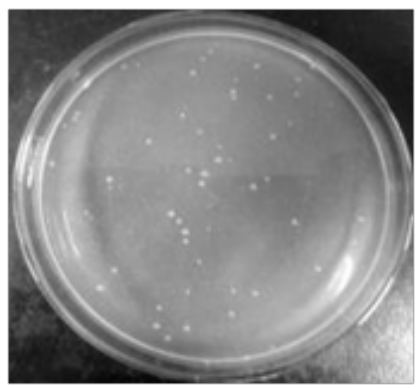

(A)

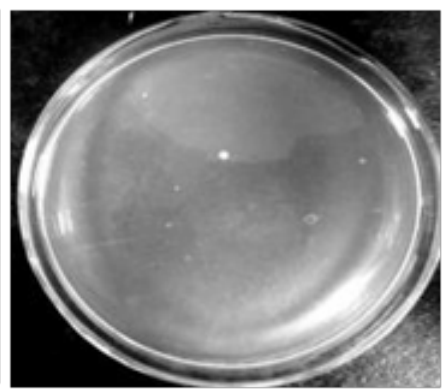

(B)

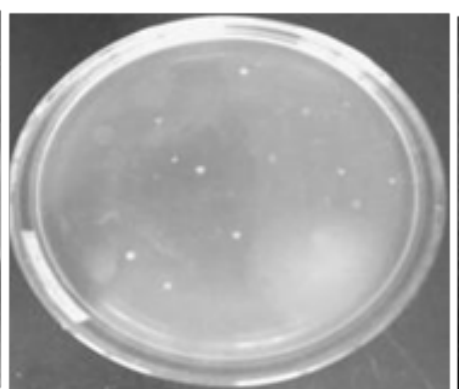

(C)

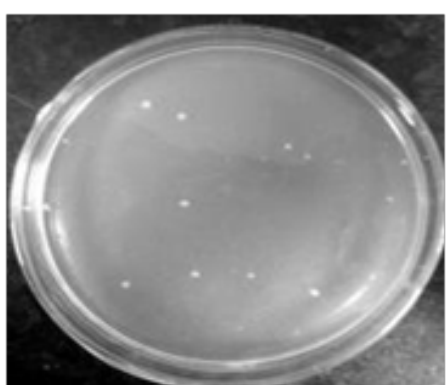

(D)

Figure I The photos of Escherichia coli colony: (A) control fabric, (B) PET-DTY with ACs, (C) DTY, and (D) spun yarn.

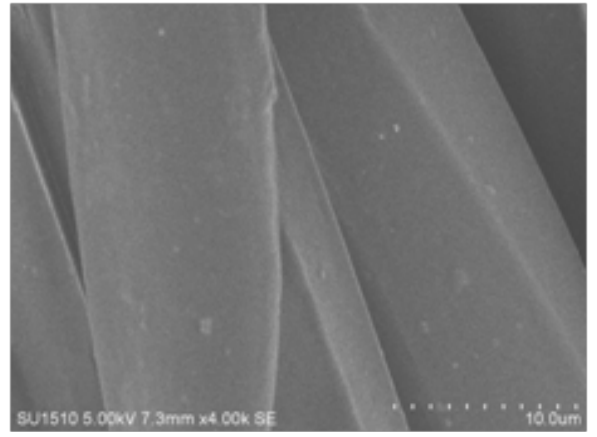

(A)

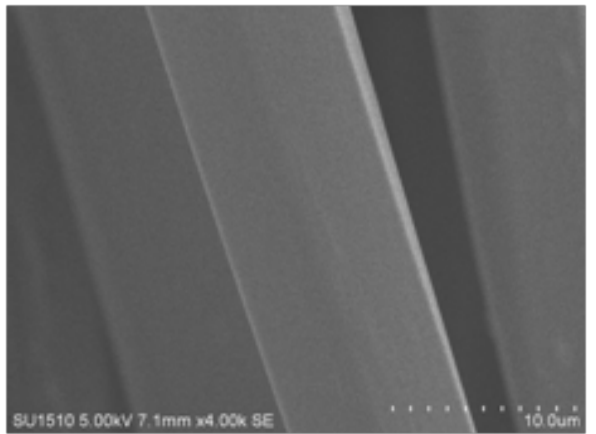

(B)

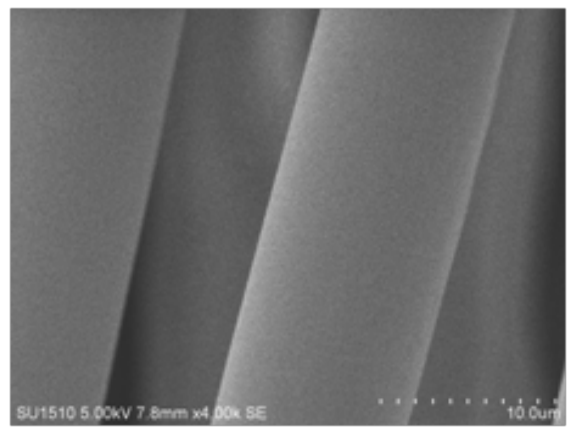

(C)

Figure 2 SEM images for PET fibres: (A) DTY+ AC, (A) DTY and (C) Spun yarn.

\section{Shape and tensile strength of PET-DTY and spun yarn}

The appearance photos of PET-DTY with and without ACs, and spun yarn were examined in Figure 4. The colour of PET-DTY with AC (Figure 4, middle) is darker than that of DTY without AC (Figure 4, upper), and the hairs cannot be observed on both of them. At the bottom of Figure 4, the hairs cover the surface of spun yarn.

For the AC dopant in PET-DTY, there is a moderate $13.2 \%$ reduction in breaking strength as shown in Table 3. As for the elongation at break, it shows 22.8 \%reduction of DTY with AC than that of DTY. The spun yarn shows the worst tensile properties as expected.

\section{Thermal-wet comfort of mattress fabric}

Heat conductivity, wettability, moisture permeability are important factors of evaluating the hot-wet comfortability of a fabric.

The heat transfer properties of fabrics are expressed by the items of the heat preservation ratio, thermal conductivity coefficient and CLO value. The behaviours of various fabrics are different in condition of perspiration. The DTY fabric with AC and spun yarn has better thermal insulation as shown in Table 4. The AC surface shows microporous, which can contain the still air to keep warm. The PET spun yarn surface has short and random fibre hairs, which can restrain air penetrating in and out to preserve the heat.
The DTY sample with AC is proved to have better wettability than that of DTY and spun yarn from Figure 5. The microporous AC has high specific surface area, which benefits the water transport.

From Figure 6, there is a little incrementation but not significant differences in the water vapour transmission rate of mattress fabrics of PET-DTY with AC and without AC compared with that of spun yarn. The reason may be that the surface hairs and short fibre length of spun yarn prevent the moisture from penetrating.

\section{Pilling property of mattress fabric}

Figure 7 shows photos of mattress fabric composed of PET-DTY with AC (Figure 7A), DTY (Figure 7B) and spun yarn (Figure 7C) after pilling. Obviously, from Figure 7, PET-DTY with AC shows no apparent difference with DTY, while the dense fuzzing and pilling covers the fabric of spun yarn.

Table 5 displays the pilling grades assigned to mattress fabrics composed of DTY with and without AC, and PET spun yarn. The samples of DTY with AC and without AC show high pilling rate (3.5), and both of them show an improvement of two grades compared to that of the spun yarn. This may be because the DTY with and without $\mathrm{AC}$ have the non circular cross-section, which reduces the fabric pilling propensity compared to circular one of spun yarn (Figure 2C). The spun yarns are hairier than the other two (Figure 4), which may allow easy exposure of raised fibre ends to abrading forces. 
Table 3 The tensile properties of PET-DTY and spun yarn

\begin{tabular}{llll}
\hline Sample & $\begin{array}{l}\text { Breaking } \\
\text { strength, cN }\end{array}$ & $\begin{array}{l}\text { Elongation } \\
\text { at break, \% }\end{array}$ & $\begin{array}{l}\text { Breaking } \\
\text { time, s }\end{array}$ \\
\hline DTY with AC & 597.4 & 19.99 & 4.77 \\
DTY & 688.6 & 25.89 & 6.17 \\
Spun yarn & 568.2 & 11.54 & 2.81 \\
\hline
\end{tabular}

Table 4 Warmth retention property of three mattress fabrics

\begin{tabular}{llll}
\hline Sample & $\begin{array}{l}\text { Heat preservation } \\
\text { ratio, \% }\end{array}$ & $\begin{array}{l}\text { Thermal } \\
\text { conductivity } \\
\text { coefficient }\end{array}$ & CLO value \\
\hline DTY+ AC & 32.77 & 31.91 & 0.21 \\
DTY & 28.02 & 38.68 & 0.17 \\
Spun yarn & 35.8 & 27.64 & 0.24 \\
\hline
\end{tabular}

Table 5 Pilling grade assigned to mattress fabrics of PET-DTY with and without CCP, PET spun yarn

\begin{tabular}{llllllll}
\hline \multirow{2}{*}{ Material } & \multicolumn{7}{c}{ Numbers of rubs } \\
\cline { 2 - 7 } & $\mathbf{1 2 5}$ & $\mathbf{5 0 0}$ & $\mathbf{1 0 0 0}$ & $\mathbf{2 0 0 0}$ & $\mathbf{3 0 0 0}$ & $\mathbf{4 0 0 0}$ & $\mathbf{5 0 0 0}$ \\
\hline $\begin{array}{l}\text { DTY } \\
\text { with AC }\end{array}$ & 5 & 5 & 4.5 & 4 & 3.5 & 3.5 & 3.5 \\
DTY & 5 & 5 & 4.5 & 4.5 & 4 & 3.5 & 3.5 \\
$\begin{array}{l}\text { Spun } \\
\text { yarn }\end{array}$ & $3-4$ & 3 & 2.5 & I-2 & I & I & I \\
\hline
\end{tabular}

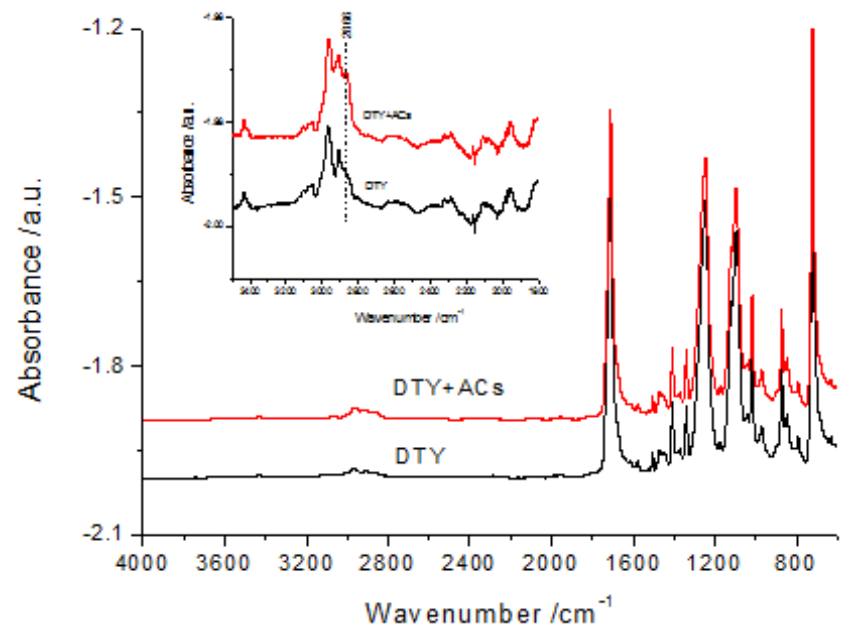

Figure 3 FTIR spectra of PET-DTY with and without AC.

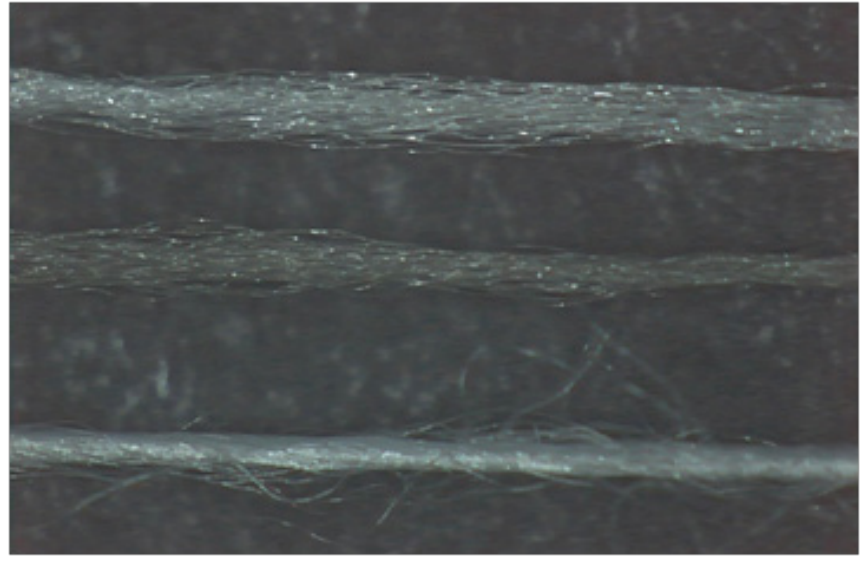

Figure 4 Representative illustrations of PET-DTY DTY without AC (upper), with AC (middle), and spun yarn (bottom).

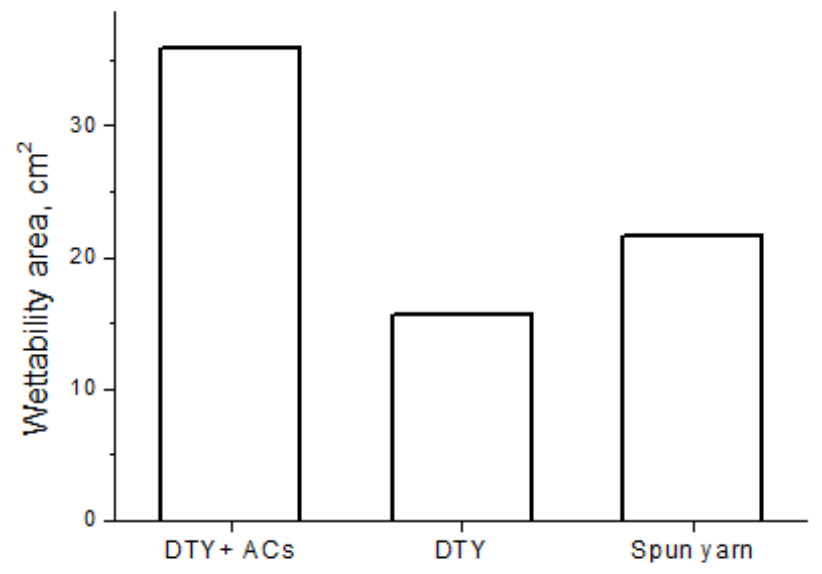

Fabric sambles

Figure $\mathbf{5}$ Wettability of three mattress fabrics.

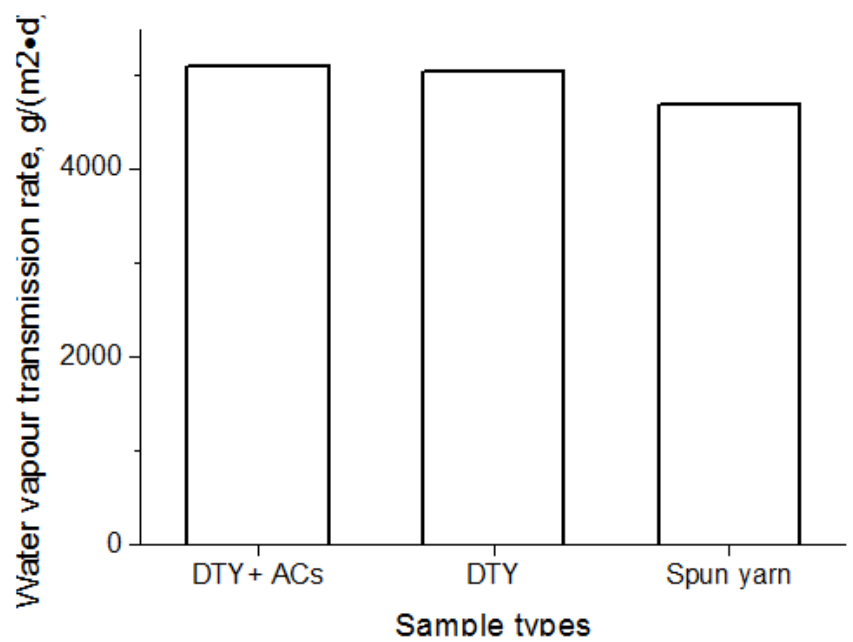

Figure 6 Moisture permeability of three types of mattress fabrics. 


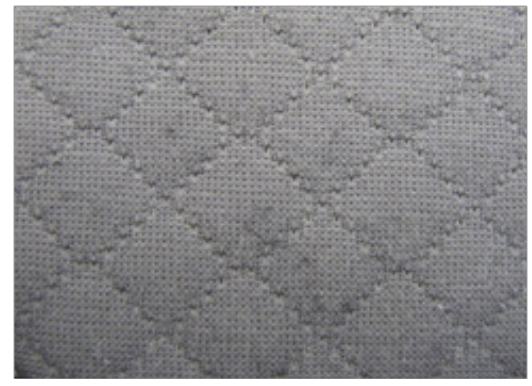

(A)

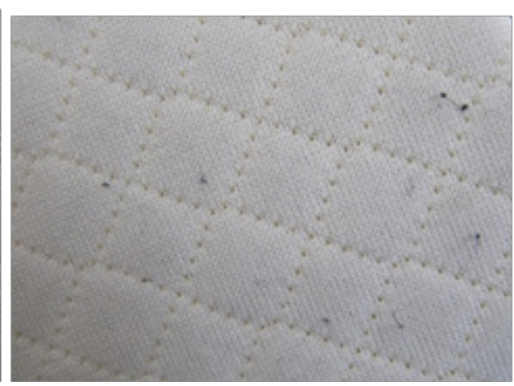

(B)

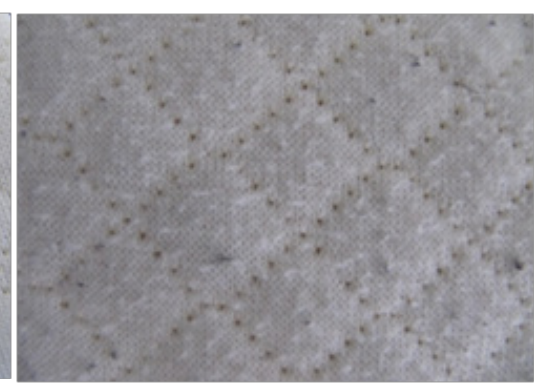

(C)

Figure 7 Camera photos of pills distribution of mattress fabrics after 3,000 rubs: (A) DTY with AC, (B) DTY, and (C) spun yarn.

\section{Conclusion}

The spinning process for to the PET fibres with coffee carbon particles is a promising technology for the antibacterial activity of weft-knitted mattress fabrics. Higher antibacterial rate $93.1 \%$ has been achieved. The microporous AC with high specific surface area benefits the water transport and moisture penetration. The non circular cross-section restricts the inner fibres protruding and reduced the entanglement of end fibres on the mattress surface during the rubs. Thus, the pilling propensity of fabric is reduced. There are no apparent changes in the chemical structures of PET-DTY with or without AC. The dopant AC does not affect the tensile property of PET-DTY.

\section{Acknowledgements}

The authors are thankful to A Project Funded by the Priority Academic Program Development of Jiangsu Higher Education Institutions (PAPD).

\section{Conflict of interest}

Author declares there is no conflict of interest in publishing the article.

\section{References}

1. Akasaka H, Takahata T, Toda I, et al. Hydrogen storage ability of porous carbon material fabricated from coffee bean wastes. Int $J$ Hydrogen Energy. 2011;36(1):580-5.
2. Ma X, Ouyang F. Adsorption properties of biomass-based activated carbon prepared with spent coffee grounds and pomelo skin by phosphoric acid activation. Appl Surf Sci. 2013;268:566-70.

3. Marsh H, Rodriguez-Reinoso F. Activated Carbon. 1st ed. New York: Elsevier; 2006. 554 p.

4. Goncalves M, Guerreiro CM, Oliveira CAL, et al. Micro mesoporous activated carbon from coffee husk as biomass waste for environmental applications. Waste Biomass Valor. 2013;4(2):395-400.

5. Goncalves M, Guerreiro MC, Alves de Oliveira LC, et al. A friendly environmental material: Iron oxide dispersed over activated carbon from coffee husk for organic pollutants removal. J Environ Manage. 2013;127:206-11.

6. Tashima D, Hamasuna Y, Mishima D, et al. Microporous activated carbons from used coffee grounds for application to electric double-layer capacitors. Ieej T Electr Electr. 2014;9(4):343-50.

7. Sekirifa M L, Cherraye R, Hadj-Mahammed M, et al. Chemical activation of an activated carbon prepared from coffee residue. Energy Procedia. 2014;50:393-400. 